Pesq. Vet. Bras. 35(5):409-416, maio 2015 DOI: 10.1590/S0100-736X2015000500004

\title{
Deficiências minerais em vacas em lactação da bacia leiteira do município de Rondon do Pará, estado do Pará ${ }^{1}$
}

\author{
Henrique A. Bomjardim² ${ }^{2}$ Carlos M.C. Oliveira², José Alcides S. Silveira² ${ }^{2}$ Natália S. \\ Silva $^{3}$, Marcos D. Duarte ${ }^{2}$, Kelson C.F. Faial ${ }^{4}$, Marilene F. Brito ${ }^{5}$ e José D. Barbosa ${ }^{2 *}$
}

\begin{abstract}
Bomjardim H.A., Oliveira C.M.C., Silveira J.A.S., Silva N.S., Duarte M.D., Faial K.C.F., Brito M.F. \& Barbosa J.D. 2015. [Mineral deficiencies in lactating cows from the dairy basin of Rondon do Pará, Brazil.] Deficiências minerais em vacas em lactação da bacia leiteira do município de Rondon do Pará, estado do Pará. Pesquisa Veterinária Brasileira 35(5):409-416. Faculdade de Medicina Veterinária, Instituto de Medicina Veterinária, Campus de Castanhal, Universidade Federal do Pará, Rodovia BR-316 Km 61, Castanhal, PA 68741-740, Brazil. E-mail: diomedes@ufpa.br

A study of mineral deficiencies was performed in lactating cows from dairy herds belonging to 13 farms of Rondon do Pará, state of Pará, Brazil. We determined the levels of phosphorus (P) in the bone and levels of copper (Cu), cobalt (Co), selenium (Se) and zinc (Zn) in the liver of 47 dairy cows in lactation 2 . The samples were collected by means of biopsies taken on the top third of the 12th rib of the right side and the caudal edge of the caudate liver lobe, respectively. The herds were composed of crossbred (Holstein x Zebu) cattle, kept in extensive production system on Brachiaria brizantha cv. Marandu pasture and received mineral supplementation. The mineral mixture on 12 farms were commercial type, called "full" and the owners added a quantity of salt above the manufacturer's recommendations on ten farms. The mineral mixtures were provided in troughs without covering on seven farms and on other eight, providing of the mineral mix was not performed daily. On 11 farms there were clinical histories consistent with mineral deficiencies in livestock, and placenta retention and osteophagia were the most frequently reported ones. The results of chemical analysis showed deficiency of P on five farms, of Co on three farms, of Se on nine farms and of $\mathrm{Zn}$ on ten farms. It is concluded that the mineral supplementation performed on the farms do not supply the daily demands of P, Se and Co, based on the estimated daily consumption of $30 \mathrm{~g}$ of $\mathrm{NaCl}$ per animal; the little adequate or inadequate troughs for proper supplementation, as well as inconstant supply of mineral mixtures contribute to the deficiency of one or more minerals.
\end{abstract}

INDEX TERMS: Bone and liver biopsy, mineral deficiencies, dairy cattle.

\footnotetext{
${ }^{1}$ Recebido em 16 de março de 2015.

Aceito para publicação em 25 de março de 2015.

${ }^{2}$ Faculdade de Medicina Veterinária, Instituto de Medicina Veterinária, Campus de Castanhal, Universidade Federal do Pará (UFPA), Rodovia BR316 Km 61, Castanhal, PA 68741-740, Brasil. *Autor para correspondência: diomedes@ufpa.br

${ }^{3}$ Universidade Federal Rural da Amazônia, Campus Paragominas, Rodovia PA-256, Bairro Nova Conquista, Paragominas, PA 68627-451, Brasil.

${ }^{4}$ Laboratório de Toxicologia "Edilson Brabo", Seção de Meio Ambiente (SAMAM), Instituto Evandro Chagas, Rodovia BR-316 Km 7, Levilândia, Ananindeua, PA 67030-000, Brasil.

${ }^{5}$ Departamento de Epidemiologia e Saúde Pública, Instituto de Veterinária (IV), Universidade Federal Rural do Rio de Janeiro (UFRRJ), Seropédica, RJ 23890-000, Brasil.
}

RESUMO.- Realizou-se um estudo das deficiências minerais em vacas em lactação de rebanhos leiteiros pertencentes a 13 propriedades da bacia leiteira do município de Rondon do Pará, estado do Pará. Foram determinados os níveis de fósforo (P) no osso, e os níveis de cobre ( $\mathrm{Cu}$ ), cobalto (Co), selênio (Se) e zinco (Zn) no fígado de 47 vacas leiteiras no 2 o terço da lactação. Estas amostras foram coletadas por meio de biópsias realizadas no terço superior da $12^{\mathrm{a}}$ costela do lado direito e no bordo caudal do lobo caudado do fígado, respectivamente. Os rebanhos eram formados por animais mestiços (Holandes x Zebu), mantidos em sistema de produção extensivo em pastos de Brachiaria brizantha cv Marandu e recebiam suplementação mineral. A mistu- 
ra mineral em 12 propriedades era do tipo comercial, dita "completa", acrescida de quantidades de $\mathrm{NaCl}$ acima do recomendado pelos fabricantes em dez propriedades. Em sete propriedades as misturas minerais eram fornecidas em cochos sem cobertura e em oito, o fornecimento da mistura mineral não era realizado diariamente. Em 11 propriedades, havia históricos clínicos condizentes com deficiências minerais nos rebanhos. Nessas fazendas a retenção de placenta e a osteofagia foram as alterações mais relatadas. Após as análises minerais observou-se deficiência de P em cinco propriedades, de Co em três propriedades, de Se em nove propriedades e de $\mathrm{Zn}$ em dez propriedades. Conclui-se que ocorre a deficiência de P, Co, Se e Zn; a suplementação mineral realizada na maioria das propriedades não atendeu as exigências diárias de $\mathrm{P}$, Se e Co, baseadas no consumo estimado de $30 \mathrm{~g}$ de $\mathrm{NaCl} / \mathrm{animal} /$ dia; os cochos pouco adequados ou inadequados para a suplementação, assim como o fornecimento inconstante das misturas minerais possivelmente contribuíram para a deficiência de um ou mais minerais.

TERMOS DE INDEXAÇÃO: Biópsia óssea e hepática, deficiências minerais, rebanhos leiteiros.

\section{INTRODUÇÃO}

Os minerais são nutrientes importantes na alimentação dos animais, assim como os carboidratos e as proteínas, pois participam de várias vias metabólicas e exercem funções importantes na reprodução, manutenção do crescimento, metabolismo energético, resposta imune, entre outras atividades metabólicas necessárias ao desempenho produtivo (Wilde 2006, Lamb et al. 2008). Em quantidades adequadas, os alimentos são capazes de fornecer os minerais para suprir as necessidades diárias dos animais, mas em muitas regiões os alimentos são pobres em determinados elementos ou os contém em proporções desequilibradas, o que impede o desempenho normal dos animais (Tokarnia et al. 2010).

Segundo Tokarnia et al. (2000), as carências minerais estão ligadas a certas áreas geográficas e quando acentuadas, podem ser responsáveis pela pobreza geral que existe em determinadas regiões, onde a população depende principalmente da criação de gado. As deficiências minerais podem ocorrer de forma severa, com perturbações mais ou menos características, ou de forma leve, com sinais não específicos, como desenvolvimento lento, problemas de fertilidade, baixo rendimento da carcaça e pouca produção de leite. Deficiências leves ou moderadas também causam prejuízos econômicos sérios, porque reduzem a produtividade dos animais e podem predispor a outras doenças.

No Brasil, as deficiências minerais, diferindo apenas nos elementos e na intensidade, ocorrem em todas as regiões e as deficiências dos macro ou microminerais específicos causam perdas econômicas à pecuária nacional, que muitas vezes inviabiliza a criação de animais em determinadas áreas, caso não ocorra a suplementação dos minerais limitantes (Tokarnia et al. 2000). No estado do Pará, as deficiências de fósforo, cobalto e cobre são comuns e, às vezes, muito severas em bovinos e bubalinos (Barbosa et al. 2005).
O estado do Pará possui o quinto maior rebanho bovino do país e ocupa a segunda colocação na produção e produtividade de leite da região norte, com 643 milhões e 637 mil litros, respectivamente. A mesorregião do sudeste paraense, composta por 39 municípios, destaca-se por ser responsável por 79\% da produção de leite do estado (IBGE 2012). Nessa região, a produção de leite transformou-se numa importante atividade econômica, constituindo hoje o segmento comercial mais expressivo para a agricultura familiar. Contudo, a produção de leite é proveniente de sistemas que exploram vacas não especializadas, utilizam pastagens mal manejadas, com severa restrição de proteína e energia para os animais no período de seca. Outro fator limitante é a falha na suplementação mineral, que geralmente é realizada de forma inadequada (Oliveira CMC, comunicação pessoal). Por isso, este trabalho tem por objetivo realizar um estudo das deficiências minerais em vacas em lactação da bacia leiteira do município de Rondon do Pará, a partir de dosagens químicas no tecido ósseo e hepático, coletados por meio de biópsias.

\section{MATERIAL E MÉTODOS}

Área de estudo. 0 levantamento das deficiências minerais foi realizado em rebanhos bovinos da bacia leiteira do município de Rondon do Pará, que é formada pelos municípios de Rondon do Pará e Abel Figueiredo, no estado do Pará e pelo município de Cidelândia, no estado do Maranhão (Figura 1). Esses municípios possuem sua produção leiteira baseada em pequenas e médias propriedades e abastecem as empresas que estão instaladas no município de Rondon do Pará.

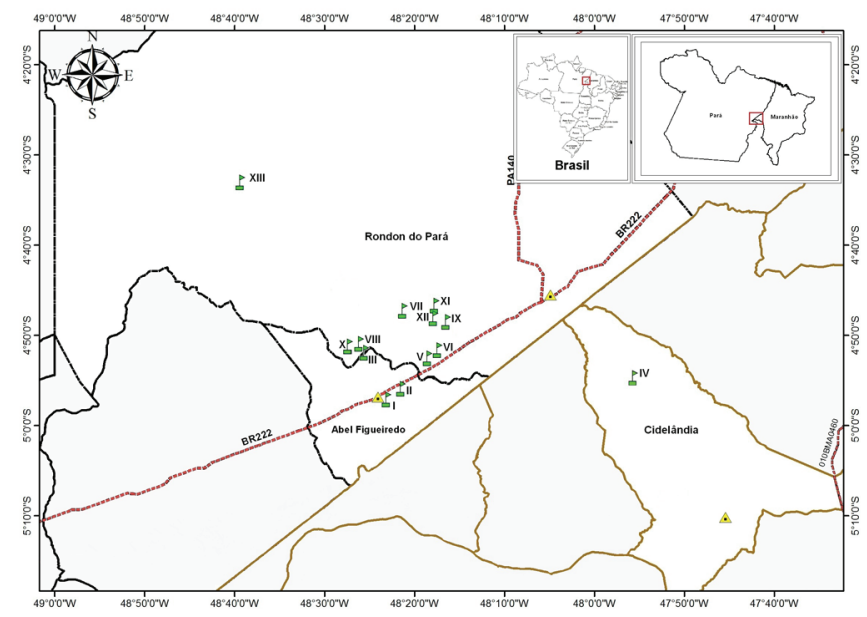

Fig.1. Bacia leiteira do município de Rondon do Pará, formada pelos municípios de Rondon do Pará, Abel Figueiredo (estado do Pará) e Cidelândia (estado do Maranhão) e distribuição das propriedades (I a XIII) entre os municípios. $\stackrel{p}{*}$ - propriedades estudadas; - - limites de municípios no estado do Pará; limites entre municípios no estado do Maranhão; - - sede dos municípios; - rodovias. Prop. I: S0457.142’ W048²3.052'; Prop. II: S0455.949' W048²1.477'; Prop. III: S0451.775' W048²6.532'; Prop. IV: S04'54.738' W047055.616'; Prop. V: S0453.930' W048 ${ }^{\circ} 18.018^{\prime}$; Prop. VI: S04 $52.717^{\prime}$ W048¹7.536'; Prop. VII: S04²7.286' W048²1.247’; Prop. VIII: S04 $49.751^{\prime}$ W048 $26.400^{\prime}$; Prop. IX: S04º $48.532^{\prime}$ W048 $16.459^{\prime}$; Prop. X: S04 ${ }^{\circ} 1.256^{\prime}$ W048 $27.369^{\prime}$; Prop. XI: S04 $46.701^{\prime}$ W048 $17.729^{\prime}$; Prop. XII: S0448.127' W048¹7.861'; Prop. XIII: S04³3.051' W048³9.321'. 
A bacia leiteira do município de Rondon do Pará está inserida na mesorregião do sudeste paraense (Rondon do Pará e Abel Figueiredo) e na mesorregião do oeste maranhense (Cidelândia). 0

\section{Quadro 1. Distribuição das propriedades por municípios, quantidade total de vacas em lactação e quantidade de amostras de osso e fígado coletadas nas propriedades da bacia leiteira do município de Rondon do Pará}

\begin{tabular}{lccc}
\hline Município/ & Vacas em lactação & \multicolumn{2}{c}{ Amostras coletad } \\
\cline { 3 - 4 } Propriedades & & Osso & Fígad \\
\hline Abel Figueiredo, PA & & & \\
I & 34 & 4 & 4 \\
II & 11 & 2 & 2 \\
III & 40 & 4 & 4 \\
Cidelândia, MA & & & \\
IV & 92 & 6 & 6 \\
Rondon do Pará, PA & & & \\
V & 67 & 5 & 5 \\
VI & 15 & 2 & 2 \\
VII & 25 & 3 & 3 \\
VIII & 15 & 2 & 2 \\
IX & 28 & 3 & 3 \\
X & 49 & 5 & 5 \\
XI & 24 & 3 & 3 \\
XII & 35 & 5 & 5 \\
XIII & 40 & 3 & 3 \\
Total & 475 & 47 & 47
\end{tabular}

clima insere-se na categoria de equatorial superúmido, tipo Am, da classificação de Köppen, no limite de transição para o tipo Aw. Possui temperaturas médias anuais de $26,35^{\circ} \mathrm{C}$, com médias máxima em torno de $32,01^{\circ} \mathrm{C}$, e mínima de $22,71^{\circ} \mathrm{C}$. A umidade relativa é elevada, com oscilações entre a estação chuvosa (novembro a maio) e a seca (junho a outubro), de 100 a 52\%, e média de 78\%. 0 índice pluviométrico anual fica em torno de $2.000 \mathrm{~mm}$ (Rondon 2009).

Propriedades, animais e período de coleta. Foram selecionadas 13 propriedades localizadas na bacia leiteira do município de Rondon do Pará. 0 critério utilizado para a seleção dessas propriedades foi a produção leiteira diária e a aceitação dos proprietários em realizar o estudo. Em cada propriedade, foram coletadas cerca de sete a $28 \%$ de osso e fígado das vacas em lactação (Quadro 1).

As coletas foram realizadas durante o final do período chuvoso. Em 12 propriedades as coletadas foram feitas no mês de junho de 2010 (propriedades de I a XII) e em uma propriedade a coleta ocorreu no mês de julho de 2011 (propriedade XIII). Durante as coletas, foi aplicado um questionário objetivo com o intuito de verificar o manejo da suplementação mineral, assim como o aparecimento de manifestações clínicas que poderiam estar relacionadas com deficiências minerais no rebanho.

Coleta das amostras. As amostras de osso foram coletadas por meio de biópsias realizadas no bordo cranial do terço superior da $12^{\text {a }}$ costela do lado direito utilizando-se uma furadeira automatizada de impacto modelo GSR 14,4 VE-2 profissional acoplada a uma serra copo modelo Starret $25 \mathrm{~mm}$ com guia suporte A01-Mandril 3/8. Para a realização destas biópsias, os animais

\section{Quadro 2. Propriedades, média de escore de condição corporal (ECC), média da produção leiteira, tipo de mistura mineral (MM), diluição da MM com o NaCl (sal branco), frequência de administração da MM, tipos de cochos utilizados nas propriedades e histórico clínico condizentes com deficiências minerais relatados nos rebanhos bovinos estudados da bacia leiteira do município de Rondon do Pará}

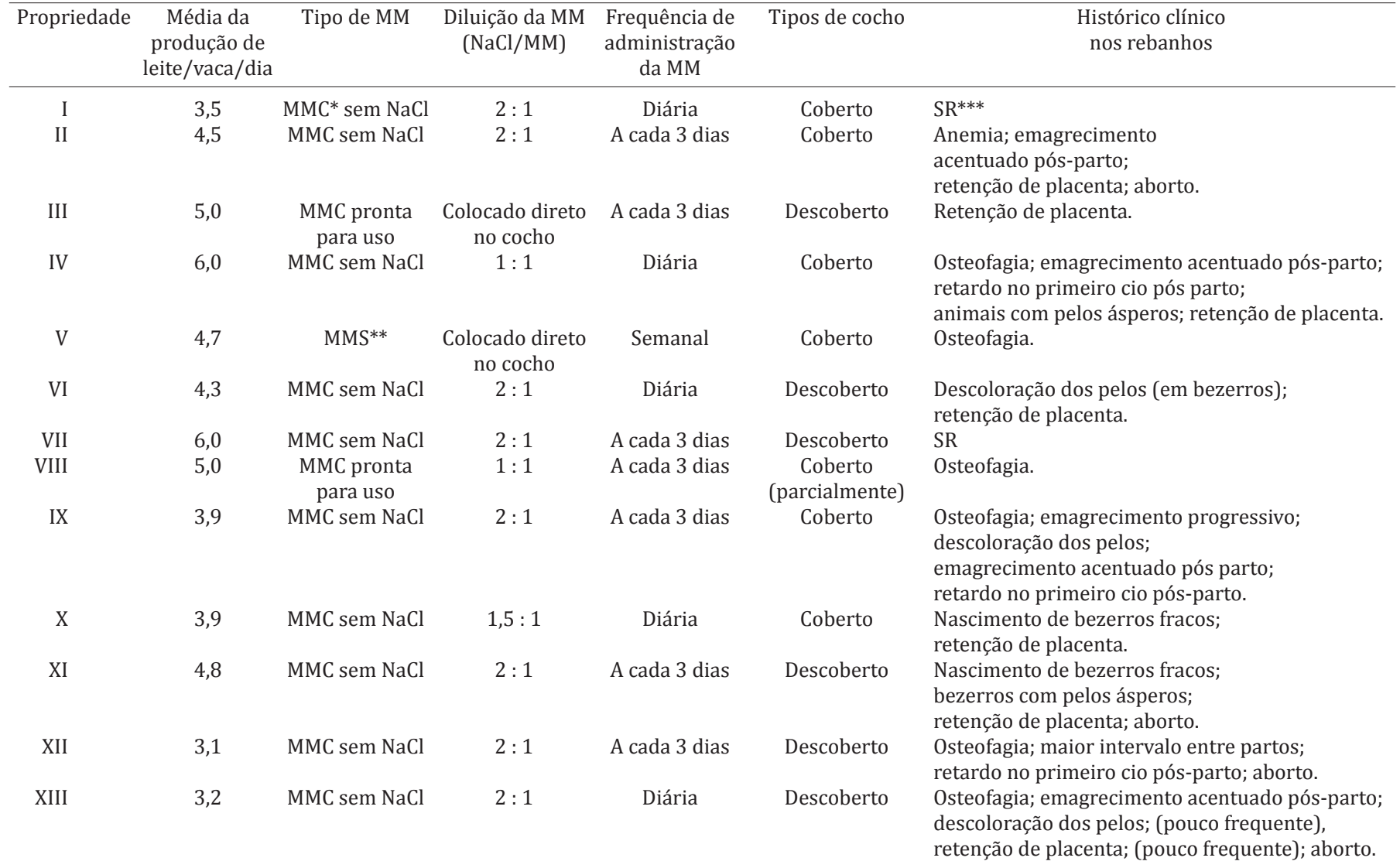


foram inicialmente sedados com cloridrato de xilazina a $2 \%$ na dose de $0,1 \mathrm{mg} / \mathrm{kg}$ de peso vivo por via intramuscular e contidos fisicamente em decúbito lateral esquerdo.

Na região do terço superior da $12^{\text {a }}$ costela do lado direito, foi realizada a tricotomia e a lavagem com água e sabão neutro, seguida de anestesia local do tipo infiltrativa intramuscular e subcutânea utilizando-se $40 \mathrm{~mL}$ de cloridrato de lidocaína a $\%$. Posteriormente, realizou-se uma segunda lavagem com água e sabão neutro da área tricotomizada e a devida assepsia utilizando-se álcool iodado a $10 \%$.

Foi realizada uma incisão de aproximadamente $10 \mathrm{~cm}$ de comprimento sobre a pele correspondente à $12^{\text {a }}$ costela e a dissecção do tecido subcutâneo para exposição da costela; foram retirados dois fragmentos de tecido ósseo do bordo cranial. Com a retirada das amostras de tecido ósseo, o peritônio foi exposto e realizou-se a incisão do mesmo, e expôs-se o bordo caudal do lobo caudado do fígado. Este foi tracionado com uma pinça do tipo atraumática modelo Doyan para a retirada de cerca de $5 \mathrm{~g}$ de fígado. A pinça foi deixada no local até a sutura do peritônio estar quase totalmente finalizada para provocar a hemostasia do órgão.

Depois de realizada as biópsias, o peritônio foi suturado com fio de catgut simples $\mathrm{n}^{\mathrm{o}} 0$ e o tecido subcutâneo com fio de nylon no $0,50 \mathrm{~mm}$ em padrão contínuo simples. Por fim, foi realizada a aproximação da pele com fio de nylon $n \times 0,80 \mathrm{~mm}$ em padrão de sutura tipo Wolf. Os animais foram tratados com oxitetraxiclina na dose única de $20 \mathrm{mg} / \mathrm{kg}$ de peso vivo, flunixin meglumine na dose de $1,1 \mathrm{mg} / \mathrm{kg}$ de peso vivo e unguento tópico.
As amostras de osso e fígado foram armazenadas em sacos plásticos limpos, previamente identificados, mantidas em caixa isotérmica com gelo reciclável e posteriormente congeladas a $-20^{\circ} \mathrm{C}$ até a realização das análises químicas dos minerais.

Dosagens químicas de osso. As análises químicas foram realizadas no Laboratório de Toxicologia da Seção de Meio Ambiente do Instituto Evandro Chagas (SAMAM/IEC/SVS/MS).

Para as análises, os tecidos ósseos foram descongelados à temperatura ambiente e removido todo o tecido mole e material medular. Posteriormente, as amostras foram pesadas em balança analítica, secas em estufa a $105^{\circ} \mathrm{C}$ durante $12 \mathrm{~h}$ e desengorduradas com éter etílico no extrator de gordura Goldfish durante $48 \mathrm{~h}$. Secou-se novamente a $105^{\circ} \mathrm{C}$ por $12 \mathrm{~h}$ em estufa, após, as amostras foram pesadas, calcinadas em mufla a $600^{\circ} \mathrm{C}$ durante $12 \mathrm{~h}$ e trituradas em gral e pistilo para a obtenção das cinzas.

Para a determinação do $\mathrm{P}$ no osso, as cinzas foram pesadas entre 0,250 a $0,260 \mathrm{~g}$ e colocadas em tubo de digestão de teflon (modelo Xpress). Em seguida, foram adicionados $3 \mathrm{~mL}$ de ácido nítrico $\left(65 \% \mathrm{~m}^{-1} \mathrm{v}^{-1}\right), 1 \mathrm{~mL}$ de ácido clorídrico $\left(30 \% \mathrm{~m} . \mathrm{v}^{-1}\right)$ e $1 \mathrm{~mL}$ de peróxido de hidrogênio $\left(30 \% \mathrm{~m} \cdot \mathrm{v}^{-1}\right)$. As amostras ficaram em repouso durante $12 \mathrm{~h}$ para uma pré-digestão, foram levadas ao forno micro-ondas (Modelo MARSXpress-CEM ${ }^{\circledR}$ ) para a digestão final e aquecidas em quatro etapas com $800 \mathrm{~W}$ de potência com duração de 10 min para cada etapa.

Após a digestão, as amostras foram colocadas em tubos de polipropileno e ajustadas ao volume final de $50 \mathrm{~mL}$. Para determinação do Íon fosfato $\left(\mathrm{PO}_{4}{ }^{3-}\right)$, foram retirados aproximadamen-

\section{Quadro 3. Quantidade de $\mathrm{NaCl}$ (sal branco), macro e microminerais obtidos em um quilo da mistura mineral (MM) fornecida às vacas em lactação das 13 propriedades estudadas da bacia leiteira do município de Rondon do Pará}

\begin{tabular}{|c|c|c|c|c|c|c|c|c|c|c|c|c|c|c|}
\hline \multirow[t]{3}{*}{ Propriedades } & \multirow[t]{3}{*}{$\mathrm{NaCl}(\mathrm{g})$} & \multicolumn{13}{|c|}{ Quantidade de minerais / kg da MM* } \\
\hline & & \multicolumn{5}{|c|}{ Macrominerais (g) } & \multicolumn{8}{|c|}{ Microminerais (mg) } \\
\hline & & $\mathrm{Na}$ & $\mathrm{Ca}$ & $\mathrm{P}$ & $\mathrm{S}$ & $\mathrm{Mg}$ & Co & $\mathrm{Cu}$ & $\mathrm{I}$ & Mn & Se & $\mathrm{Zn}$ & $\mathrm{Fe}$ & $\mathrm{F}$ \\
\hline I & 667 & 246,8 & 70,7 & 53,3 & 8,3 & 5,3 & 66,7 & $1.000,0$ & 66,7 & 466,7 & 10,0 & $1.666,7$ & - & 638,3 \\
\hline II & 667 & 246,8 & 71,7 & 56,7 & 4,0 & 5,3 & 103,3 & $1.166,7$ & 93,3 & $1.213,3$ & 9,0 & $2.500,0$ & 653,3 & - \\
\hline III & 297 & 110,0 & 180,0 & 44,0 & 0,6 & 13 & 30,0 & 750,0 & 60,0 & 585,0 & 12,0 & $2.470,0$ & 350,0 & - \\
\hline IV & 500 & 185,0 & 104,0 & 80,0 & 7,5 & - & 300,0 & $1.350,0$ & 135,0 & $2.310,0$ & 25,0 & $2.310,0$ & - & - \\
\hline $\mathrm{V}$ & 500 & 185,0 & - & 97,5 & - & - & 126,0 & 875,0 & - & 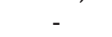 & 36,0 & $1.010,0$ & - & - \\
\hline VI & 667 & 246,8 & 130,0 & 80,0 & 15,0 & 2,5 & 125,0 & $1.500,0$ & 90,0 & 175,0 & 22,5 & 504,0 & - & - \\
\hline VII & 667 & 246,8 & 69,7 & 54,3 & 4,0 & 4,2 & 103,3 & $1.166,7$ & 93,3 & $1.213,3$ & 10,7 & $3.000,0$ & 653,3 & 543,3 \\
\hline VIII & 573 & 212,0 & 107,5 & 85,0 & 140,0 & 6,0 & 155,0 & $1.750,0$ & 980,0 & - & 37,5 & $1.820,0$ & - & - \\
\hline IX & 667 & 246,8 & 69,3 & 53,3 & 5,0 & - & 200,0 & 900,0 & 90,0 & $1.540,0$ & 16,7 & $1.540,0$ & - & - \\
\hline $\mathrm{X}$ & 600 & 222,0 & 86,0 & 68,0 & 4,8 & 6,4 & 124,0 & $1.400,0$ & 112,0 & $1.456,0$ & 10,8 & $3.000,0$ & 784,0 & - \\
\hline XI & 667 & 246,8 & 71,7 & 56,7 & 4,0 & 5,3 & 103,3 & $1.166,7$ & 93,3 & $1.213,3$ & 9,0 & $2.500,0$ & 653,3 & - \\
\hline XII & 667 & 246,8 & 65,0 & 50,0 & 6,7 & 13,3 & 133,3 & 666,7 & 66,7 & 666,7 & 33,3 & 400,0 & - & - \\
\hline XIII & 667 & 246,8 & 69,7 & 54,3 & 4,0 & 4,2 & 103,3 & $1.166,7$ & 93,3 & $1.213,3$ & 10,7 & $3.000,0$ & 653,3 & 543,3 \\
\hline
\end{tabular}

* Calcularam-se estas quantidades a partir das informações obtidas nos rótulos das embalagens das misturas minerais ofertadas aos animais.
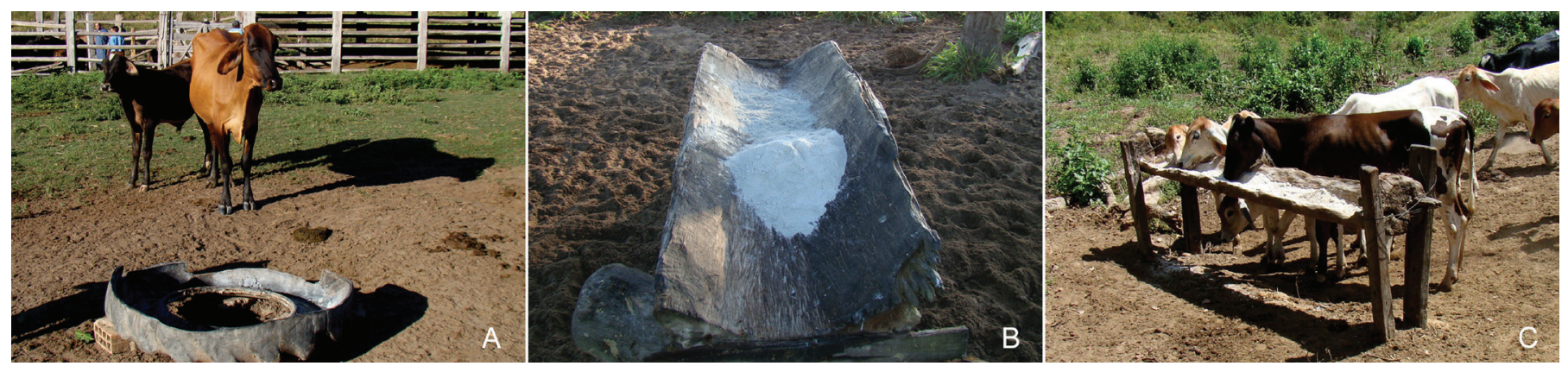

Fig.2. Cochos sem cobertura utilizados para a suplementação mineral de rebanhos bovinos da bacia leiteira do município de Rondon do Pará. (A) Cocho produzido de pneu de trator da Propriedade I, Abel Figueiredo, PA. (B) Cocho de madeira da Propriedade XI, Rondon do Pará, PA. (C) Cocho de madeira da Propriedade XII, Rondon do Pará, PA. 
te $5 \mathrm{~mL}$ dessa solução para ser analisada por cromatografia de íons, em sistema ICS 2000 DUAL (THERMO SCINTIFIC-DIONEX, USA).

Dosagens químicas de fígado. As amostras de fígado foram seccionadas em fatias finas utilizando-se navalhas limpas e de aço inoxidável, armazenadas em tubos de polipropileno de $50 \mathrm{~mL}$ e submetidas ao processo de liofilização. Para esse processo, as amostras de fígado, congeladas a $-20^{\circ} \mathrm{C}$, foram colocadas em bandejas e colocadas em aparelho automatizado LIOTOP ${ }^{\circledR}$ (modelo L101), previamente ligado por um período de 10 min a uma temperatura de $-55^{\circ} \mathrm{C}$, as quais permaneceram por um período de $24 \mathrm{~h}$ para a secagem completa das amostras. Posteriormente, as amostras foram trituradas em grau e pistilo.

Para a determinação dos minerais $\mathrm{Cu}, \mathrm{Co}$, Se e $\mathrm{Zn}$, as amostras de fígado trituradas foram pesadas entre 0,250 a $0,260 \mathrm{~g}$ e colocadas em tubo de digestão de teflon (modelo Xpress). Em seguida, foi adicionado $3 \mathrm{~mL}$ de ácido nítrico $\left(65 \% \mathrm{~m} \cdot \mathrm{v}^{-1}\right), 1 \mathrm{~mL}$ de ácido clorídrico $\left(30 \% \mathrm{~m} \cdot \mathrm{v}^{-1}\right)$ e $1 \mathrm{~mL}$ de peróxido de hidrogênio P.A. (30\% $\left.\mathrm{m} . \mathrm{v}^{-1}\right)$. As amostras ficaram em repouso durante $2 \mathrm{~h}$ para uma pré-digestão e digeridas por 50 min em sistema fechado por radiação de micro-ondas (MARSXpress, CEM Corp. Matthews, NC, USA). Após a digestão, os minerais $\mathrm{Cu}$, Co e $\mathrm{Zn}$ foram analisados pela técnica de Espectrometria de Emissão Ótica com Plasma Induzido (ICP OES) no equipamento ICP-OES (Vista-MPX CCD simultâneo, axial da VARIAN) em sistema de amostragem automático (SPS-5). Já o Se foi determinado pela técnica de Espectometria de Emissão Ótica com acoplamento de Geração de Hidretos (HG-ICP OES) no equipamento modelo iCAP 6000-CCD simultâneo (Thermo Scientific, Madison, USA), configuração axial e equipado com sistema de amostragem automático (CETAC-ASX 520). O controle das condições operacionais do ICP OES foi realizada com o software iTEVA. Os brancos analíticos foram preparados pelos mesmos procedimentos sem a adição das amostras de fígado (Nomura et al. 2005).

Consumo de mistura mineral. Foi realizada uma análise nas misturas minerais ofertadas aos animais das propriedades estudadas, a partir das informações contidas nos rótulos das embalagens, a fim de estimar o consumo diário por animal de $\mathrm{P}, \mathrm{Co}, \mathrm{Cu}, \mathrm{Se}$ e Zn. Essa análise foi estimada a partir do consumo médio de $30 \mathrm{~g}$ de $\mathrm{NaCl} /$ animal/dia, segundo Tokarnia et al. (2000).

\section{RESULTADOS}

Nas 13 propriedades avaliadas da bacia leiteira do município de Rondon do Pará, os animais eram criados em sistema de produção extensivo em pastos formados predominantemente por Brachiaria brizantha cv Marandu e eram suplementados com misturas minerais comerciais. Em todas as propriedades os animais recebiam água à vontade.

Os rebanhos leiteiros das propriedades estudadas eram formados por animais mestiços (Holandês x Zebu); as vacas em lactação, no período das coletas, apresentavam boa condição corporal (escore corporal médio de 3, em uma escala de 1 a 5) e a produção de leite média dos rebanhos era de 3,1 a 6,0 litros de leite/vaca/dia (Quadro 2).

A suplementação mineral fornecida aos animais em 12 propriedades estudadas eram misturas minerais comerciais (MMC) do tipo "completa" ( $\mathrm{Na}, \mathrm{Cl}, \mathrm{Ca}, \mathrm{P}, \mathrm{S}, \mathrm{Mg}$, $\mathrm{Co}, \mathrm{Cu}$, I, Mn, Se, Zn, Fe e F) não específicas para vacas em lactação. $\mathrm{Na}$ propriedade $\mathrm{V}$, era fornecida mistura mineral seletiva (MMS) aos animais ( $\mathrm{Na}, \mathrm{Cl}, \mathrm{P}, \mathrm{Co}, \mathrm{Cu}, \mathrm{Se}$ e $\mathrm{Zn}$ ) (Quadro 3).

Em nove propriedades, as $\mathrm{MMC}$ sem $\mathrm{NaCl}$ eram acrescidas dessa substância na proporção de 2:1 (2 de $\mathrm{NaCl}$ para 1 de MMC) ou 1,5:1, contudo as recomendações do fabricante indicavam que as MMC deveriam ser misturadas na mesma proporção, ou seja, 1 saco $(25 \mathrm{~kg})$ de MMC para 1 saco $(25 \mathrm{~kg})$ de $\mathrm{NaCl}$. Em duas propriedades, a MMC pronta para uso era misturada com $\mathrm{NaCl}$ em uma proporção de 1:1. As demais propriedades seguiam as recomendações dos fabricantes (Quadros 2 e 3).

\section{Quadro 4. Valores de P no osso e de Co, Cu, Se e Zn no fígado de vacas em lactação da bacia leiteira do município de} Rondon do Pará

\begin{tabular}{|c|c|c|c|c|c|c|}
\hline \multirow[t]{2}{*}{ Propriedades } & \multirow[t]{2}{*}{ Animal } & \multirow{2}{*}{$\begin{array}{l}\text { Osso } \\
\mathrm{P}(\%)\end{array}$} & \multicolumn{4}{|c|}{ Fígado } \\
\hline & & & Co & $\begin{array}{l}\mathrm{Cu} \\
(\mathrm{mg}\end{array}$ & $\begin{array}{r}\mathrm{Se} \\
\left(\mathrm{g}^{-1}\right)\end{array}$ & $\mathrm{Zn}$ \\
\hline \multirow[t]{4}{*}{ I } & 1 & 18,1 & 0,903 & 151,4 & 1,06 & 130,4 \\
\hline & 2 & 18,0 & $<\mathrm{LD}^{* *}$ & 437,6 & 2,04 & 71,5 \\
\hline & 3 & 18,3 & 0,319 & 279,0 & 0,93 & 187,4 \\
\hline & 4 & 19,3 & 0,119 & 194,2 & 0,87 & 81,2 \\
\hline \multirow[t]{2}{*}{ II } & 5 & 18,9 & 0,837 & 323,2 & 0,99 & 187,9 \\
\hline & 6 & 19,1 & 0,153 & 195,6 & 1,02 & 58,0 \\
\hline \multirow[t]{4}{*}{ III } & 7 & 19,2 & 0,526 & 149,1 & 0,74 & 64,3 \\
\hline & 8 & 18,3 & 0,370 & 203,4 & 0,32 & 138,0 \\
\hline & 9 & 18,1 & 0,719 & 357,1 & 0,80 & 144,3 \\
\hline & 10 & 18,1 & 0,385 & 130,5 & 0,76 & 82,2 \\
\hline \multirow[t]{5}{*}{ IV } & 11 & 17,4 & 1,802 & 173,2 & 1,47 & 266,4 \\
\hline & 12 & 16,7 & 1,085 & 244,5 & 1,32 & 84,1 \\
\hline & 13 & 17,7 & 1,141 & 562,0 & 1,19 & 157,4 \\
\hline & 14 & 17,3 & 0,635 & 429,3 & 0,77 & 128,7 \\
\hline & 15 & 17,4 & 0,878 & 345,3 & 1,63 & 97,2 \\
\hline \multirow[t]{6}{*}{ V } & 16 & 16,5 & 0,615 & 59,4 & 0,73 & 90,9 \\
\hline & 17 & 18,5 & 0,414 & 154,1 & 0,75 & 55,4 \\
\hline & 18 & 19,8 & 0,459 & 287,4 & 1,23 & 77,8 \\
\hline & 19 & 18,0 & 1,057 & 193,1 & 1,00 & 99,2 \\
\hline & 20 & 17,6 & 0,607 & 391,0 & 1,14 & 193,7 \\
\hline & 21 & 19,5 & 0,350 & 200,4 & 0,65 & 97,7 \\
\hline \multirow[t]{2}{*}{ VI } & 22 & 16,6 & 0,793 & 258,9 & 0,79 & 164,9 \\
\hline & 23 & 19,0 & 0,863 & 654,1 & 1,22 & 324,0 \\
\hline \multirow[t]{3}{*}{ VII } & 24 & 18,4 & $<\mathrm{LD}^{* *}$ & 180,2 & 0,84 & 74,2 \\
\hline & 25 & 16,8 & 0,668 & 306,3 & 0,67 & 136,0 \\
\hline & 26 & 17,4 & 0,011 & 120,1 & 0,66 & 64,6 \\
\hline \multirow[t]{2}{*}{ VIII } & 27 & 18,5 & 0,719 & 335,2 & 1,30 & 140,7 \\
\hline & 28 & 18,6 & 0,440 & 270,6 & 1,18 & 111,8 \\
\hline \multirow[t]{3}{*}{ IX } & 29 & 19,0 & 0,121 & 335,7 & 0,93 & 112,7 \\
\hline & 30 & 17,5 & 0,669 & 234,8 & 0,70 & 93,5 \\
\hline & 31 & 18,7 & $<\mathrm{LD}^{* *}$ & 551,4 & 1,22 & 87,6 \\
\hline \multirow[t]{5}{*}{ X } & 32 & 17,7 & 0,731 & 491,8 & 2,15 & 230,0 \\
\hline & 33 & 18,5 & 1,339 & 242,2 & 1,45 & 206,0 \\
\hline & 34 & 17,9 & 0,494 & 367,1 & 1,16 & 208,1 \\
\hline & 35 & 17,5 & 0,070 & 141,3 & 1,27 & 109,9 \\
\hline & 36 & 17,7 & 0,509 & 225,0 & 1,22 & 159,7 \\
\hline \multirow[t]{3}{*}{ XI } & 37 & 17,5 & 0,768 & 144,0 & ND & 62,1 \\
\hline & 38 & 18,2 & 0,286 & 273,3 & ND & 112,1 \\
\hline & 39 & 17,2 & 0,068 & 423,8 & ND & 132,5 \\
\hline \multirow[t]{5}{*}{ XII } & 40 & 17,7 & 1,439 & 203,9 & ND & 246,1 \\
\hline & 41 & 16,7 & 1,149 & 286,6 & ND & 126,8 \\
\hline & 42 & 16,4 & 0,779 & 662,9 & ND & 137,8 \\
\hline & 43 & 16,6 & 0,599 & 106,0 & ND & 72,3 \\
\hline & 44 & 16,6 & 0,763 & 60,5 & ND & 157,2 \\
\hline \multirow[t]{3}{*}{ XIII } & 45 & 16,9 & 0,088 & 121,8 & 0,73 & 89,2 \\
\hline & 46 & ND & 0,365 & 187,9 & 0,34 & 93,6 \\
\hline & 47 & 16,4 & 0,290 & 168,8 & 0,52 & 70,1 \\
\hline ma & & $17^{\mathrm{a}}$ & $0,12^{\mathrm{b}}$ & $50^{\mathrm{b}}$ & $1^{c}$ & $100^{t}$ \\
\hline
\end{tabular}

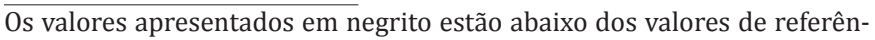
cia. *Valores normais para a espécie bovina, abaixo destes são deficientes, ${ }^{* *}$ Valor abaixo do limite de detecção (LD) do aparelho, ${ }^{* * *}$ ND = não determinado. ${ }^{\text {a }}$ Riet-Correa \& Timm (2007), ${ }^{\mathrm{b}}$ Underwood \& Suttle (1999), ' Dirksen et al. (2005). 
Quadro 5. Consumo estimado de P, Co, Cu, Se e $\mathrm{Zn}$ baseado na ingestão média de $30 \mathrm{~g}$ de $\mathrm{NaCl} /$ animal/dia das propriedades da bacia leiteira do município de Rondon do Pará

\begin{tabular}{lcccccccccccccc}
\hline Minerais & $\begin{array}{c}\text { Exigências/ } \\
\text { animal/dia* }\end{array}$ & I & II & III & IV & V & VI & VII & VIII & IX & X & XI & XII & XIII \\
\hline P (g) & 6 & $\mathbf{2 , 4}$ & $\mathbf{2 , 5}$ & $\mathbf{4 , 4}$ & $\mathbf{4 , 8}$ & $\mathbf{5 , 8}$ & $\mathbf{3 , 6}$ & $\mathbf{2 , 4}$ & $\mathbf{4 , 4}$ & $\mathbf{2 , 4}$ & $\mathbf{3 , 4}$ & $\mathbf{2 , 5}$ & $\mathbf{2 , 2}$ & $\mathbf{2 , 4}$ \\
Co (mg) & $5-10$ & $\mathbf{3 , 0}$ & $\mathbf{4 , 6}$ & $\mathbf{3 , 0}$ & 18,0 & 7,6 & 5,6 & $\mathbf{4 , 6}$ & 8,1 & 9,0 & 6,2 & $\mathbf{4 , 6}$ & $\mathbf{6 , 0}$ & $\mathbf{4 , 6}$ \\
Cu (mg) & 50 & $\mathbf{4 5 , 0}$ & 52,5 & 75,8 & 81,0 & 52,5 & 67,5 & 52,5 & 91,6 & $\mathbf{4 0 , 5}$ & 70,0 & 52,5 & $\mathbf{3 0 , 0}$ & 52,5 \\
Se (mg) & $1-3$ & $\mathbf{0 , 4}$ & $\mathbf{0 , 4}$ & 1,2 & 1,5 & 2,2 & 1,0 & $\mathbf{0 , 5}$ & 2,0 & $\mathbf{0 , 7}$ & $\mathbf{0 , 5}$ & $\mathbf{0 , 4}$ & 1,5 & $\mathbf{0 , 5}$ \\
Zn (mg) & $43,5-87$ & 75,0 & 112,4 & 249,5 & 138,6 & 60,6 & $\mathbf{2 2 , 7}$ & 134,9 & 95,3 & 69,3 & 150,0 & 112,4 & $\mathbf{1 8 , 0}$ & 134,9
\end{tabular}

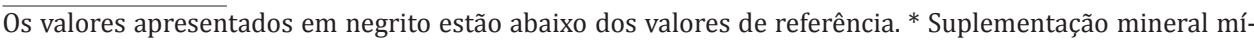
nima para evitar os efeitos negativos das deficiências minerais em bovinos em crescimento (Tokarnia et al. 2000).

Em todas as propriedades, as misturas minerais eram ofertadas aos animais em cochos instalados nas pastagens. Em sete delas, estes cochos não eram cobertos (Fig.2) e em oito propriedades, o fornecimento da mistura mineral nos cochos não era realizado diariamente (Quadro 2).

Em 11 propriedades, foram relatados pelos proprietários sinais clínicos condizentes com deficiências minerais. A retenção de placenta e a osteofagia foram as alterações mais relatadas (Quadro 2).

Os valores de $\mathrm{P}$ no osso e de $\mathrm{Co}, \mathrm{Cu}$, Se e $\mathrm{Zn}$ no fígado dos animais avaliados estão apresentados por propriedade no Quadro 4. Já o consumo estimado diário de P, Co, Cu, Se e Zn por animal está demonstrado no Quadro 5. Os níveis deficientes ou subdeficientes e o consumo estimado diário de minerais por animal abaixo do recomendado estão apresentados em negrito.

Pelas análises químicas dos minerais foram observadas deficiência de $\mathrm{P}$ (em pelo menos um animal) nos animais das propriedades IV, VI, VII, XII e XIII, de Co nas propriedades I, VII e IX, de Se nas propriedades I, II, III, IV, V, VI, VII, IX e XIII e de Zn nas propriedades I, II, III, IV, V, VII, IX, XI, XII e XIII. Não foi observada deficiência de $\mathrm{Cu}$ nos animais das propriedades estudadas (Quadro 4).

\section{DISCUSSÃO}

Este é o primeiro estudo sobre o diagnóstico de deficiências minerais realizado em vacas em lactação de rebanhos da bacia leiteira do município de Rondon do Pará e da região Norte do Brasil. Pelas análises dos minerais no osso e no fígado, históricos clínicos condizentes com deficiências minerais nos rebanhos e suplementação mineral realizada de forma inadequada por parte dos produtores, determinou-se que vacas em lactação da bacia leiteira do município de Rondon do Pará possuem deficiência de P, Se, Zn e Co.

Em 12 propriedades, a mistura mineral utilizada era do tipo "completa" e os proprietários adicionavam quantidades de $\mathrm{NaCl}$ acima do recomendado pelos fabricantes. Segundo Malafaia et al. (2014), esta prática, considerada inadequada, aumenta a concentração de $\mathrm{NaCl}$ e consequentemente reduz o consumo de certos minerais, mesmo que estejam em quantidades suficientes na mistura total. Na região em estudo, essa é uma tentativa utilizada pelos proprietários para tentar reduzir os custos com a suplementação mineral do rebanho, já que o $\mathrm{NaCl}$ é bem mais barato.

Quanto ao fornecimento da mistura mineral, em oito propriedades não era realizada de forma constante e os cochos utilizados para a suplementação mineral na maioria das propriedades estudadas eram pouco adequados ou inadequados para a suplementação mineral dos animais. Em sete propriedades, os cochos não possuíam proteção contra as chuvas. Para Peixoto et al. (2005) é importante disponibilizar a mistura mineral aos animais de forma que o consumo diário seja cerca de 30 a $50 \mathrm{~g}$ de $\mathrm{NaCl}$ por animal e também manter a qualidade da mistura mineral para que se garanta o seu consumo. Do contrário, a mistura mineral não será ingerida em quantidade necessária. Portanto, o fornecimento inconstante destas misturas minerais em cochos inadequados para a suplementação mineral foram fatores que contribuíram para a ocorrência de deficiência de um ou mais minerais nas propriedades.

Nas cinco propriedades em que ocorreu a deficiência de $\mathrm{P}$ o consumo estimado de $\mathrm{P}$ via mistura mineral estava abaixo de 6g/animal/dia. Segundo Tokarnia et al. (2000), esta é a quantidade de $\mathrm{P}$ diária necessária para que um bovino na fase de crescimento não apresente os efeitos negativos da deficiência de P. Acredita-se que, para vacas adultas não especializadas para a produção de leite, esta quantidade seja o suficiente para que não ocorra a deficiência de P nestes animais. Apesar de fornecerem a suplementação mineral aos animais, o baixo consumo estimado de P nestas misturas possivelmente favoreceu a deficiência deste elemento nos animais. Contudo, nas demais propriedades o consumo estimado de $\mathrm{P}$ estava abaixo de 6g/animal/dia e apesar disso não foi determinada a deficiência de $\mathrm{P}$ nos animais. Sugere-se que fatores ligados aos animais como pouca especialização para a produção de leite, o que diminuiria a exigência em minerais, associado à suplementação mineral durante todos os meses do ano podem ter contribuído para estes resultados.

Em relação ao Co, o limite mínimo utilizado na metodologia para a detecção deste elemento no fígado foi de 0,003ppm. Sabendo-se que níveis de Co abaixo de 0,05 ppm são valores considerados críticos, certificamos que os três animais (propriedades I, VII e IX), que não tiveram os níveis de Co detectadas pela metodologia, são deficientes neste elemento, como já determinado por Pinheiro et al. (2011).

Nas propriedades I e VII, nas quais foram observados valores deficientes em Co nos animais, o consumo estimado diário de Co por animal pela mistura mineral ofertada estava abaixo de $5 \mathrm{mg}$. De acordo com Tokarnia et al. (2000), o consumo diário de 5 a 10mg de Co pela mistura 
mineral é necessário para que os bovinos não desenvolvam deficiência deste mineral. Nas propriedades II, III, XI e XIII, não foi observada deficiência de Co, porém o consumo estimado diário de Co por animal estava abaixo de $5 \mathrm{mg}$. Provavelmente, nestas propriedades, as exigências diárias não supridas pela mistura ofertada de Co foram complementadas pela pastagem.

Nas propriedades estudadas não foram observados valores deficientes em $\mathrm{Cu}$ nas vacas em lactação, contudo dois animais apresentaram valores subdeficientes nas propriedades IV e XII, sendo que, na propriedade XII, o consumo diário estimado de $\mathrm{Cu}$ por animal estava abaixo de $50 \mathrm{mg}$, porém na propriedade IV estava acima. No entanto, Tokarnia et al. (2000) afirmam que um bovino deve ingerir $100 \mathrm{mg}$ de $\mathrm{Cu}$ por dia para não apresentar deficiência deste mineral e a metade desta quantidade $(50 \mathrm{mg})$ deve ser garantida pela suplementação mineral. Nas propriedades I e IX, em que os animais apresentaram valores normais de $\mathrm{Cu}$ no fígado, o consumo diário estimado de $\mathrm{Cu}$ por animal estava abaixo de $50 \mathrm{mg}$. Diante disto, é possível afirmar que o consumo de $\mathrm{Cu}$ ofertado na mistura mineral juntamente com o $\mathrm{Cu}$ presente nas pastagens supriu as necessidades diárias de $\mathrm{Cu}$ aos animais para que não ocorresse a deficiência deste elemento.

Na determinação de Se, Underwood \& Suttle (1999) afirmam que os animais são deficientes neste elemento quando as análises do fígado indicam níveis de Se abaixo de $0,1 \mathrm{mg} \cdot \mathrm{kg}^{-1}$. No entanto, Dirksen et al. (2005) indicam que os valores de Se no fígado acima de $1 \mathrm{mg} \cdot \mathrm{kg}^{-1}$ são normais e valores abaixo deste são deficientes. Pela metodologia utilizada, não foram obtidos níveis de Se abaixo de 0,1mg. $\mathrm{kg}^{-1}$, o menor valor de Se observado foi de $0,32 \mathrm{mg} \cdot \mathrm{kg}^{-1}$ e o maior de 2,15mg.kg-1. Porém, em sete propriedades havia histórico clínico de retenção de placenta no rebanho. Para Lucci et al. (1987), os índices de retenção de placenta em vacas são maiores sem a suplementação com Se. Por isso, o valor considerado normal de Se no fígado dos bovinos no presente estudo foi de $1 \mathrm{mg}^{\mathrm{kg}}{ }^{-1}$, os valores abaixo deste foram considerados deficientes.

Dentre as oito propriedades com animais deficientes em Se, observou-se que a mistura mineral nas propriedades I, II, VII, XI e XIII não atendia as exigências diárias de Se por animal, ou seja, o consumo estimado de Se para cada $30 \mathrm{~g}$ de $\mathrm{NaCl}$ estava abaixo de $1 \mathrm{mg}$. Para Tokarnia et al. (2000), é recomendado administrar pelas misturas minerais aos animais cerca de 1 a $3 \mathrm{mg}$ de Se/animal/dia. Com esta estimativa, é provável dizer que as pastagens são deficientes em Se e a suplementação ofertada aos animais não supre a quantidade diária de Se para que se evite a deficiência deste mineral nas vacas em lactação.

Nas propriedades I, II, III, IV, V, VII, IX, XI e XIII, em que foram observados animais com valores deficientes em $\mathrm{Zn}$, o consumo estimado diário de $\mathrm{Zn}$ por animal estava acima de $43,5 \mathrm{mg}$ e somente na propriedade XII o consumo estimado diário de Zn por animal estava abaixo deste valor. Para Tokarnia et al. (2000), são necessários acrescentar 250 a $500 \mathrm{~g}$ de sulfato de zinco a $50 \mathrm{~kg}$ de $\mathrm{NaCl}$ para não ocorrer a deficiência de $\mathrm{Zn}$ nos animais. Como o sulfato de zinco tem em média $29 \%$ de $\mathrm{Zn}$, a cada $30 \mathrm{~g}$ de $\mathrm{NaCl}$, um bovino deve- rá consumir 43,5mg de Zn. Na propriedade VI, o consumo estimado diário de Zn por animal também estava abaixo de 43,5mg, porém não foram observados animais deficientes em Zn. Estes resultados demonstram que os animais deficientes em Zn nas propriedades em estudo não estavam relacionados com o consumo de $\mathrm{Zn}$ nas misturas minerais ofertadas.

Nas propriedades II, III, V, VII, IX e XI, a suplementação não era constante e nas propriedades III, VII e XIII os cochos não eram cobertos, o que pode ter diminuído a ingestão da mistura mineral pelos animais e causado a deficiência de Zn. Porém, nas propriedades I e IV o fornecimento da mistura mineral era realizado diariamente e os cochos eram cobertos. É provável que as concentrações de Ca nas pastagens conjuntamente com o Ca ofertado nas misturas minerais, antagonizou a absorção de Zn pelos animais, como relatado por Riet-Correa (2007) e Oliveira (2014). No entanto, Brum et al. (1987) observaram baixos valores de Zn no tecido hepático de vacas de corte em lactação no pantanal Mato-grossense, resultados semelhantes a este estudo, porém a deficiência deste elemento nos animais foi atribuída à baixa concentração de $\mathrm{Zn}$ observada nas forragens.

Embora a maioria das propriedades apresentem animais com níveis deficientes de $\mathrm{Zn}$ no tecido hepático, não foi obtido histórico de animais com quadro clínico de paraqueratose pelos proprietários. Para Tokarnia et al. (2000), quadros clínicos de paraqueratose em bovinos são raros em todo o mundo e a deficiência de $\mathrm{Zn}$ ocorre na forma subclínica, com sinais clínicos inespecíficos em diversas regiões do mundo, inclusive no Brasil. Pelos baixos níveis de Zn no fígado e histórico clínico inespecíficos observados nos rebanhos em estudo, tudo indica que a deficiência de Zn nos animais ocorra de forma subclínica.

\section{CONCLUSÕES}

A partir dos resultados obtidos neste estudo é possível concluir que na bacia leiteira do município de Rondon do Pará:

Ocorre a deficiência de P, Co, Se e Zn nas propriedades estudadas;

A suplementação mineral realizada nas propriedades, na maior parte, não atende às exigências diárias de $\mathrm{P}$, Se e $\mathrm{Co}$, baseado no consumo estimado de $30 \mathrm{~g}$ de $\mathrm{NaCl} / \mathrm{animal} /$ dia;

Os cochos inadequados para a suplementação mineral, assim como o fornecimento inconstante das misturas minerais nos cochos e a adição de $\mathrm{NaCl}$ nas misturas minerais acima das recomendações dos fabricantes, possivelmente, contribuíram para a deficiência de um ou mais minerais.

Agradecimentos,- Ao Conselho Nacional de Desenvolvimento Científico e Tecnológico (CNPq) pela concessão de bolsa de mestrado e ao Instituto Evandro Chagas pelo apoio a realização das dosagens dos minerais.

\section{REFERÊNCIAS}

Barbosa J.D., Oliveira C.M.C., Duarte M.D. \& Silveira J.A.S. 2005. Doenças de búfalos na Amazônia. II Simpósio Mineiro de Buiatria, Belo Horizonte, MG. 
Brum P.A.R., Sousa J.C., Comastri Filho J.A. \& Almeida I.L. 1987. Deficiências minerais de bovinos na sub-região dos Paiaguás, no Pantanal Mato-Grossense. II. Cobre, zinco, manganês e ferro. Pesq. Agropec. Bras. 22(9/10):1049-1060.

Dirksen G., Gründer H.D. \& Stöber M. 2005. Medicina Interna y Cirurgía del Bovino. $4^{\mathrm{a}}$ ed. Inter-Médica, Buenos Aires. 632p.

IBGE 2012. Pesquisa da Pecuária Municipal. Fundação Instituto Brasileiro de Geografia e Estatística, Brasília.

Lamb G.C., Brown D.R., Larson J.E., Dahlen C.R., Dilorenzo N., Arthington J.D. \& Dicostanzo A. 2008. Effect of organic or inorganic trace mineral supplementation on follicular response, ovulation, and embryo production in superovulated Angus heifers. Anim. Reprod. Sci. 106(3/4):221-231.

Lucci C.S., Schalch E., Zanetti M.A., Pettinati R.L. \& Ostronoffi S. 1987. Selênio em bovinos de leite em Itirapina, estado de São Paulo. Pesq. Agropec. Bras. 22(6):653-656.

Malafaia P., Costa R.M., Brito M.F., Peixoto P.V., Barbosa J.D., Tokarnia C.H. \& Jürgen Döbereiner. 2014. Equívocos arraigados no meio pecuário sobre deficiências minerais e suplementação minerais em bovinos no Brasil. Pesq. Vet. Bras. 34(3):244-249.

Nomura C.S., Silva C.S., Nogueira A.R.A. \& Oliveira P.V. 2005. Bovine liver sample preparation and micro-homogeneity study for $\mathrm{Cu}$ and $\mathrm{Zn}$ determination by solid sampling electrothermal atomic absorption spectrometry. Spectrochim. Acta B 60:673-680.

Oliveira C.M.C. 2014. Diagnóstico das deficiências minerais de macro e micro minerais em búfalas (Bubalus bubalis) provenientes da Ilha de Marajó, estado do Pará. Tese de Doutorado, Universidade Federal do Pará, Belém. 69p.
Peixoto P.V., Malafaia P., Barbosa J.D., Canella C.F.C. \& Tokarnia C.H. 2005. Princípios de suplementação mineral em ruminantes. Pesq. Vet. Bras. 25(3):195-200.

Pinheiro C.P., Bomjardim H.A., Andrade S.J.T., Faial K.C.F., Oliveira C.M.C. \& Barbosa J.D. 2011. Níveis de fósforo, cobre, cobalto e zinco em bubalinos (Bubalus bubalis) na Ilha de Marajó, Estado do Pará. Pesq. Vet. Bras. 31(3):193-198.

Riet-Correa F. 2007. Carências minerais, p.223-233. In: Riet-Correa F., Schild A.L., Lemos R.A.A. \& Borges J.R.J. (Eds), Doenças de Ruminantes e Equídeos. 3aㅡ ed. Pallotti, Santa Maria.

Riet-Correa F. \& Timm C.D. 2007. Deficiência de fósforo, p.248-257. In: Riet-Correa F., Schild A.L., Lemos R.A.A. \& Borges J.R.J. (Eds), Doenças de Ruminantes e Eqüídeos. 3를. Pallotti, Santa Maria.

Rondon do Pará, 2009. Geografia: Wikipédia. (Disponível em <http://pt. wikipedia.org/wiki/Rondon_do_Par\%C3\%A1\#Geografia> Acesso em: 15 Junho 2013.)

Tokarnia C.H., Döbereiner J. \& Peixoto P.V. 2000. Deficiências minerais em animais de fazenda, principalmente bovinos em regime de campo. Pesq. Vet. Bras. 20(3):127-138

Tokarnia C.H., Peixoto P.V., Barbosa J.D., Brito M.F. \& Döbereiner J. 2010. Deficiências minerais em animais de produção. Helianthus, Rio de Janeiro, $191 \mathrm{p}$.

Underwood E.J. \& Suttle N.F. 1999. The Mineral Nutrition of Livestock. 3rd ed. CABI Publishing, Wallingford. $614 \mathrm{p}$.

Wilde D. 2006. Influence of macro and micro minerals in the peri-parturient period on fertility in dairy cattle. Anim. Reprod. Sci. 96:240-249. 